\title{
Effect of Second Sphere Interactions on the Magnetic Anisotropy of Lanthanide Single-Molecule Magnets: Electrostatic Interactions and Supramolecular Contacts
}

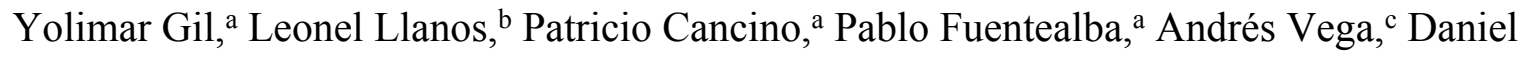
Aravena, ${ }^{\mathrm{b},{ }^{*}}$ Evgenia Spodine ${ }^{\mathrm{a},{ }^{*}}$

a Facultad de Ciencias Químicas y Farmacéuticas, Universidad de Chile, Casilla 233, Santiago, Chile.

b Departamento de Química de los Materiales, Facultad de Química y Biología, Universidad de Santiago de Chile, Casilla 40, Correo 33, Santiago, Chile.

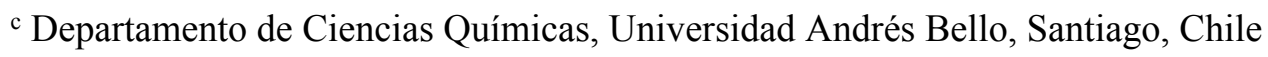


Table S1. Crystal data and structure refinement details for $\mathbf{1}$ and $\mathbf{2 .}$

\begin{tabular}{|c|c|c|}
\hline & 1 & 2 \\
\hline Chemical formula & {$\left[\mathrm{Dy}(\mathrm{N}-\mathrm{NCS})_{3}\left(\mathrm{H}_{2} \mathrm{O}\right)_{5}\right] \cdot 0.45(\mathrm{KSCN})(18 \mathrm{C} 6)$} & {$\left[\mathrm{Dy}\left(\mathrm{NO}_{3}\right)_{2}(\mathrm{~N}-\mathrm{NCS})_{3}\left(\mathrm{H}_{2} \mathrm{O}\right)\right] \cdot\left(\mathrm{H}_{2} \mathrm{O}\right)\left(\mathrm{NH}_{4}\right)_{2} 2(18 \mathrm{C} 6)$} \\
\hline$M_{\mathrm{r}}$ & 1449.57 & 1059.50 \\
\hline Crystal system, space group & Triclinic, $P \overline{1}$ & Orthorhombic, $P m n 2_{1}$ \\
\hline Temperature (K) & 293 & 293 \\
\hline$a, b, c(\AA)$ & $10.1936(7), 10.6025(6), 14.6812(9)$ & 23.783(10), 11.337(5), 8.490(4) \\
\hline$\alpha, \beta, \nu\left(^{\circ}\right)$ & $88.189(3), 79.328(3), 77.725(3)$ & $90,90,90$ \\
\hline$V\left(\AA^{3}\right)$ & $1523.57(17)$ & $2289.1(17)$ \\
\hline$Z$ & 1 & 2 \\
\hline Radiation type & Mo $K_{\alpha}$ & Mo $K_{\alpha}$ \\
\hline$\mu\left(\mathrm{mm}^{-1}\right)$ & 2.80 & 1.85 \\
\hline Crystal size (mm) & $0.42 \times 0.11 \times 0.08$ & $0.20 \times 0.14 \times 0.13$ \\
\hline$T_{\min }, T_{\max }$ & $0.448,0.746$ & $0.621,0.925$ \\
\hline \multicolumn{3}{|l|}{ No. of measured, independent } \\
\hline $\begin{array}{l}\text { and observed }[\mathrm{I}>2 \sigma(\mathrm{I})] \\
\text { reflections }\end{array}$ & $11590,5966,4859$ & $17432,4602,4197$ \\
\hline $\boldsymbol{R}_{\text {int }}$ & 0.049 & 0.062 \\
\hline$(\sin \theta / \lambda)_{\max }\left(\AA^{-1}\right)$ & 0.617 & 0.617 \\
\hline \multicolumn{3}{|l|}{ Refinemen } \\
\hline$R\left[F^{2}>2 \mathrm{~s}\left(F^{2}\right)\right], w R\left(F^{2}\right), S$ & $0.056,0.149,1.07$ & $0.033,0.068,1.03$ \\
\hline No. of reflections & 5966 & 4602 \\
\hline No. of parameters & 343 & 288 \\
\hline No. of restraints & 1 & 3 \\
\hline $\mathrm{H}$-atom treatment & $\begin{array}{l}\mathrm{H} \text { atoms treated by a mixture of } \\
\text { independent and constrained refinement }\end{array}$ & $\begin{array}{l}\mathrm{H} \text { atoms treated by a mixture of independent } \\
\text { and constrained refinement }\end{array}$ \\
\hline$\Delta \rho_{\max }, \Delta \rho_{\min }\left(\mathrm{e} \AA^{-3}\right)$ & $1.57,-1.33$ & $0.67,-0.72$ \\
\hline
\end{tabular}


Table S2. Selected bond distances $(\AA)$ and angles $\left({ }^{\circ}\right)$ for $\mathbf{1}$, and $\mathbf{2}$

\begin{tabular}{|c|c|c|c|}
\hline \multicolumn{4}{|c|}{1} \\
\hline Dy1-02w & $2.369(6)$ & Dy1-01w & 2.391(5) \\
\hline Dy1-N1 & $2.371(7)$ & Dy1-N2 & $2.421(8)$ \\
\hline Dy1-03w & $2.372(7)$ & Dy1-04w & $2.440(6)$ \\
\hline Dy1-05w & $2.382(6)$ & Dy1-N3 & $2.389(7)$ \\
\hline $\mathrm{O} 2 \mathrm{w}-\mathrm{Dy} 1-\mathrm{N} 1$ & $81.4(3)$ & N3-Dy1-O1w & $73.6(2)$ \\
\hline $02 w-D y 1-03 w$ & $76.8(3)$ & $\mathrm{O} 2 \mathrm{w}-\mathrm{Dy} 1-\mathrm{N} 2$ & $147.2(2)$ \\
\hline N1-Dy1-O3w & $74.1(3)$ & $\mathrm{N} 1-\mathrm{Dy} 1-\mathrm{N} 2$ & $75.8(3)$ \\
\hline $02 w-D y 1-05 w$ & $140.8(2)$ & $\mathrm{O} 3 \mathrm{w}-\mathrm{Dy} 1-\mathrm{N} 2$ & 118.0(3) \\
\hline N1-Dy1-05w & $115.9(3)$ & $\mathrm{O} 5 \mathrm{w}-\mathrm{Dy} 1-\mathrm{N} 2$ & $71.5(2)$ \\
\hline $03 w-D y 1-05 w$ & $75.3(2)$ & $\mathrm{N} 3-\mathrm{Dy} 1-\mathrm{N} 2$ & $79.3(3)$ \\
\hline $\mathrm{O} 2 \mathrm{w}-\mathrm{Dy} 1-\mathrm{N} 3$ & $106.5(3)$ & $\mathrm{O} 1 \mathrm{w}-\mathrm{Dy} 1-\mathrm{N} 2$ & $80.0(2)$ \\
\hline $\mathrm{N} 1-\mathrm{Dy} 1-\mathrm{N} 3$ & $142.2(3)$ & $02 w-D y 1-04 w$ & $73.6(2)$ \\
\hline $\mathrm{O} 3 \mathrm{w}-\mathrm{Dy} 1-\mathrm{N} 3$ & $143.5(3)$ & N1-Dy1-O4w & $142.5(3)$ \\
\hline O5w-Dy1-N3 & $81.5(3)$ & O3w-Dy1-04w & 73.4(3) \\
\hline $02 w-D y 1-01 w$ & $71.3(2)$ & O5w-Dy1-04w & $72.5(2)$ \\
\hline N1-Dy1-01w & 74.4(3) & N3-Dy1-O4w & $73.0(2)$ \\
\hline $03 w-D y 1-01 w$ & $137.7(2)$ & O1w-Dy1-04w & $121.0(2)$ \\
\hline $05 w-D y 1-01 w$ & $145.0(2)$ & $\mathrm{N} 2-\mathrm{Dy} 1-04 \mathrm{w}$ & 137.1(2) \\
\hline \multicolumn{4}{|c|}{2} \\
\hline Dy1-N3 & $2.363(6)$ & Dy1-07 & $2.410(6)$ \\
\hline Dy $1-N 3^{i}$ & $2.363(6)$ & Dy1-017 & $2.483(10)$ \\
\hline Dy1-N4 & $2.369(6)$ & Dy1-08 & $2.500(4)$ \\
\hline Dy1-01 & $2.377(9)$ & Dy1-08 & $2.500(4)$ \\
\hline $\mathrm{N} 3-\mathrm{Dy} 1-\mathrm{N} 3^{\mathrm{i}}$ & $154.5(3)$ & 07-Dy1-017 & $52.7(3)$ \\
\hline N3-Dy1-N4 & $82.12(15)$ & N3-Dy1-O8i & $127.19(17)$ \\
\hline $\mathrm{N} 3{ }^{\mathrm{i}}-\mathrm{Dy} 1-\mathrm{N} 4$ & $82.12(15)$ & $\mathrm{N} 3^{\mathrm{i}}-\mathrm{Dy} 1-08^{\mathrm{i}}$ & $76.42(17)$ \\
\hline N3-Dy1-O1 & $97.92(15)$ & $\mathrm{N} 4-\mathrm{Dy} 1-08^{\mathrm{i}}$ & $141.6(2)$ \\
\hline $\mathrm{N} 3{ }^{\mathrm{i}}-\mathrm{Dy} 1-\mathrm{O} 1$ & $97.92(15)$ & O1-Dy1-08i & $74.9(3)$ \\
\hline N4-Dy1-01 & $77.1(4)$ & O7-Dy1-08i & $76.60(18)$ \\
\hline N3-Dy1-07 & $88.46(14)$ & O17-Dy1-08 & $123.6(2)$ \\
\hline N3i-Dy1-O7 & $88.46(14)$ & N3-Dy1-08 & $76.42(17)$ \\
\hline N4-Dy1-07 & $134.6(4)$ & N3'-Dy1-O8 & 127.19(17) \\
\hline 01-Dy1-07 & $148.3(3)$ & N4-Dy1-O8 & 141.6(2) \\
\hline N3-Dy1-017 & $79.02(14)$ & 01-Dy1-08 & $74.9(3)$ \\
\hline N3i-Dy1-017 & $79.02(14)$ & O7-Dy1-08 & $76.60(18)$ \\
\hline N4-Dy1-017 & $81.9(4)$ & 017-Dy1-08 & $123.6(2)$ \\
\hline 01-Dy1-017 & $158.9(2)$ & O8i-Dy1-08 & $51.0(2)$ \\
\hline
\end{tabular}

Symmetry codes: i: $-x, y, z$. ii: $x,-y+1 / 2, z$.

Table S3. Hydrogen-bond geometry $\left(\AA,^{\circ}\right)$ for 2

\begin{tabular}{|c|c|c|c|c|}
\hline$D-H \cdots A$ & $D-H$ & $H \cdots A$ & $D \cdots A$ & $D-H \cdots A$ \\
\hline \multicolumn{5}{|c|}{2} \\
\hline O1-H1w…07wiii & 0.85 & 1.88 & $2.694(13)$ & 159 \\
\hline
\end{tabular}

Symmetry codes: iii: $x, y-1, z$; iv: $\mathrm{x}, y-1, z-1$; v: $-x+1,-y+1,-z+1$. 


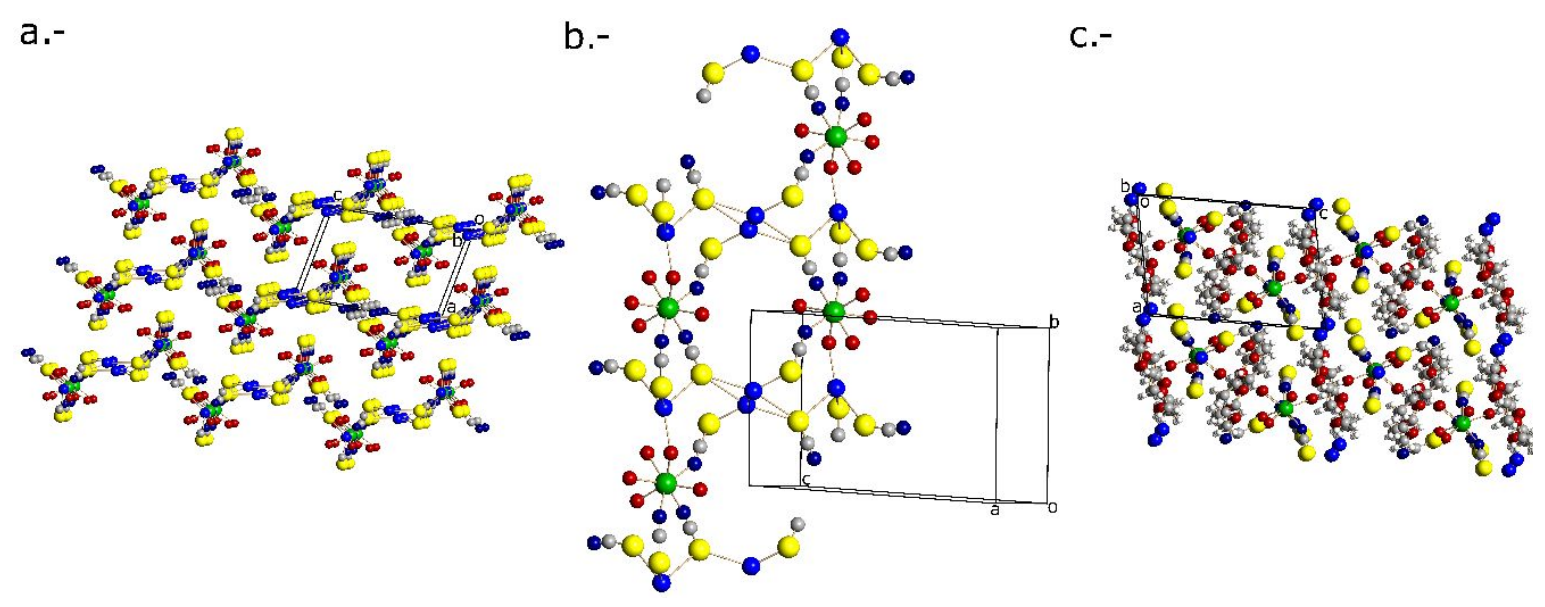

Figure S1. Packing view for the structure of $\left[\mathrm{Dy}(\mathrm{N}-\mathrm{NCS})_{3}\left(\mathrm{H}_{2} \mathrm{O}\right)_{5}\right] \cdot 0.45(\mathrm{KNCS}) \cdot(18$-crown6). a.- View along [010] and crown ether molecules omitted. b.- Approximately [100] view of chains defined by $\left[\mathrm{Dy}(\mathrm{N}-\mathrm{NCS})_{3}\left(\mathrm{H}_{2} \mathrm{O}\right)_{5}\right]$ units and the interstitial potassium ions. c.- View along [010].

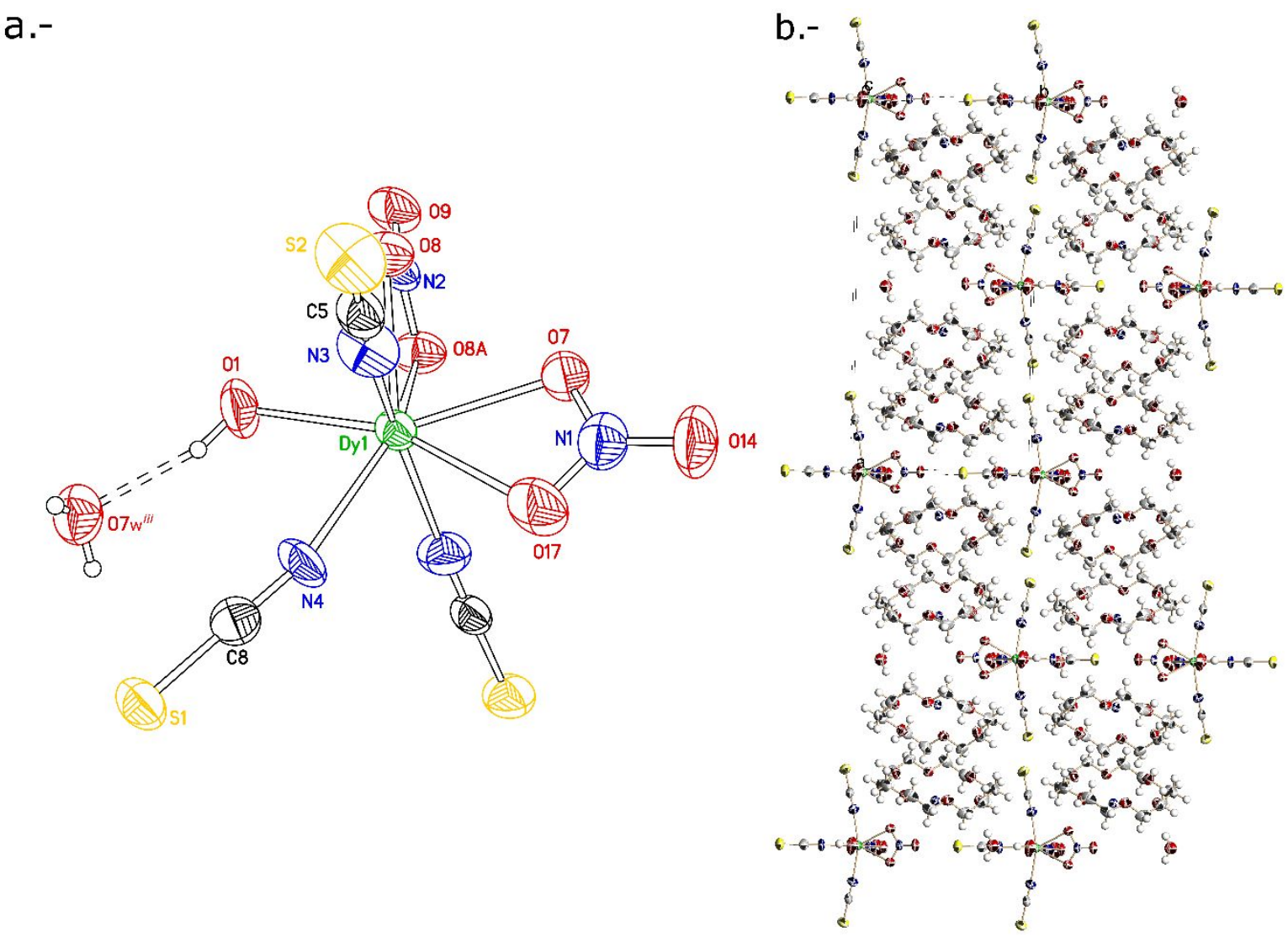

Figure S2. a.- Hydrogen bond between $\left[\mathrm{Dy}\left(\mathrm{NO}_{3}\right)_{2}(-\mathrm{N}-\mathrm{NCS})_{3}\left(\mathrm{H}_{2} \mathrm{O}\right)\right]^{2-}$ (2) and the crystallization water. b.- Packing view along [001]. 


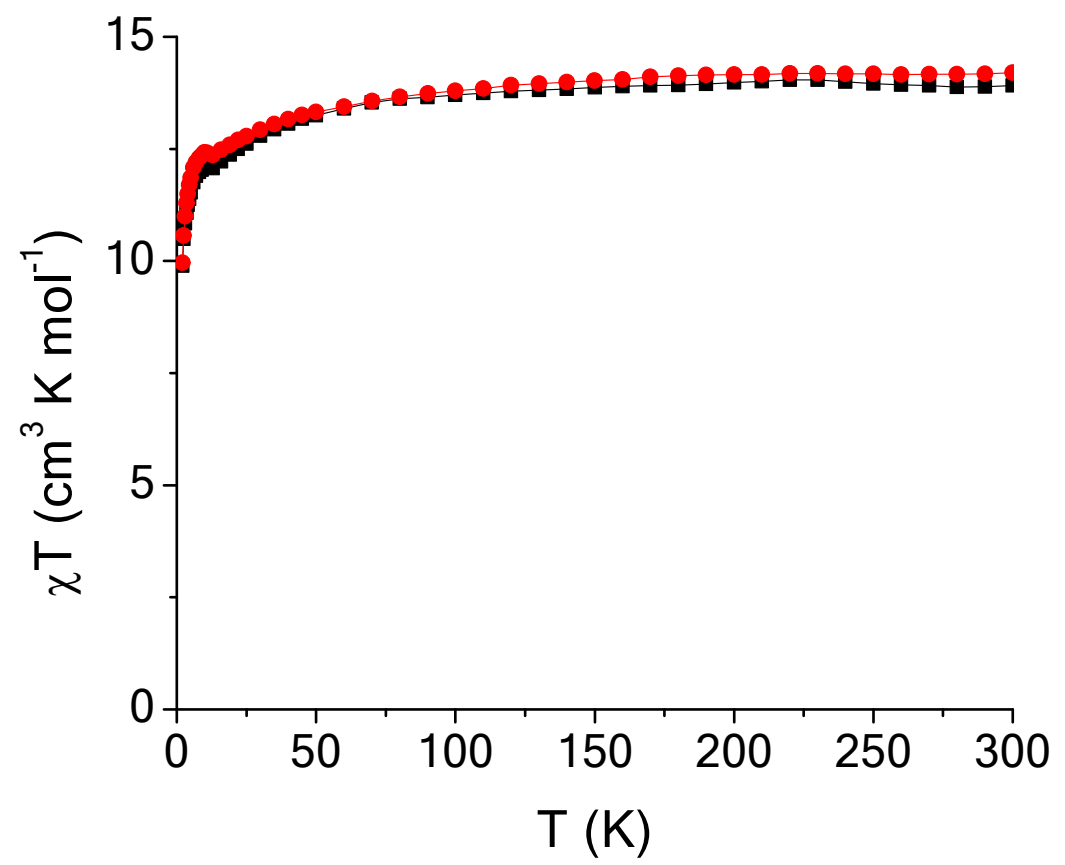

Figure S3. Temperature dependent magnetic susceptibility for $\mathbf{1}$ (black), $\mathbf{2}$ (red) at a static field of 30000 .

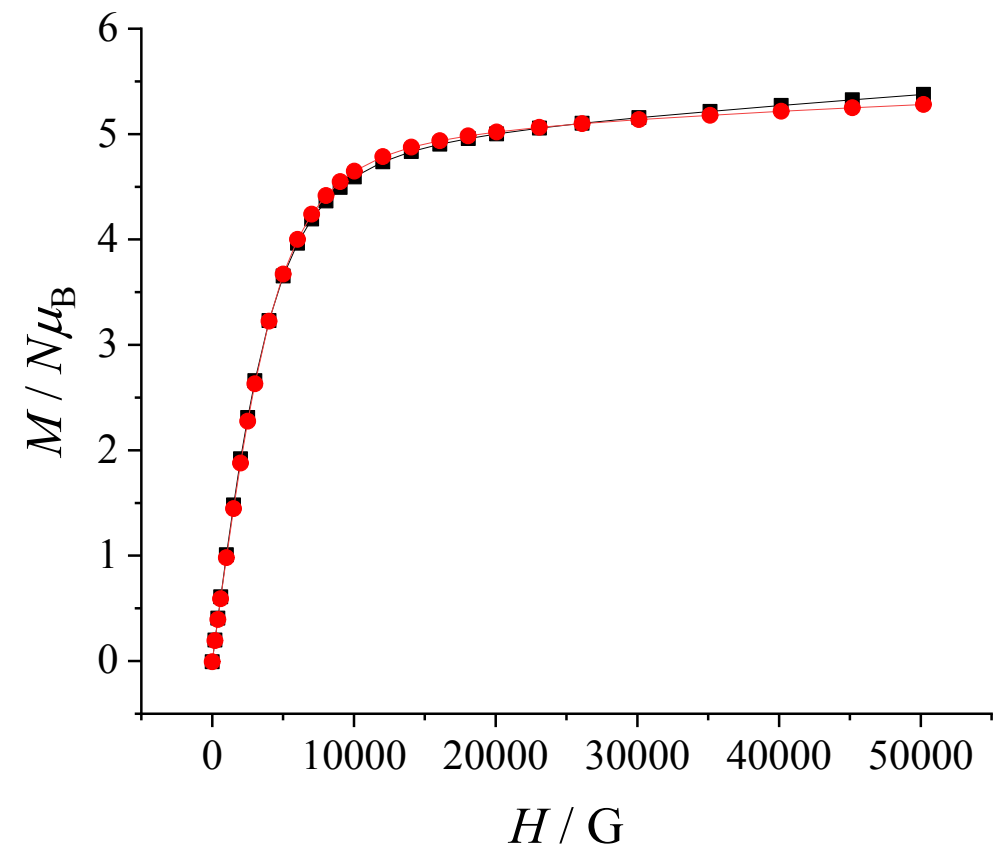

Figure S4. Field dependence of the magnetization measured at $2 \mathrm{~K}$ for $\mathbf{1}$ (black) and $\mathbf{2}$ (red). 
Table S4. Natural atomic charges for different fragments of the 'full' and 'small' models of 1 and 3.

\begin{tabular}{ccc|ccc}
\hline & $\mathbf{1}$ & & \multicolumn{3}{c}{$\mathbf{3}$} \\
& full & small & & full & small \\
\hline $\mathrm{Y}$ & 1.976 & 1.971 & $\mathrm{Y}$ & 1.848 & 1.856 \\
$\mathrm{SCN}$ & -0.839 & -0.829 & $\mathrm{NO}_{3}$ & -0.736 & -0.737 \\
$\mathrm{SCN}$ & -0.830 & -0.824 & $\mathrm{NO}_{3}$ & -0.735 & -0.733 \\
$\mathrm{SCN}$ & -0.833 & -0.827 & $\mathrm{NO}_{3}$ & -0.741 & -0.732 \\
$\mathrm{H}_{2} \mathrm{O}$ & 0.082 & 0.103 & $\mathrm{H}_{2} \mathrm{O}(\mathrm{W} 1)$ & 0.080 & 0.116 \\
$\mathrm{H}_{2} \mathrm{O}$ & 0.084 & 0.096 & $\mathrm{H}_{2} \mathrm{O}(\mathrm{W} 2)$ & 0.063 & 0.113 \\
$\mathrm{H}_{2} \mathrm{O}$ & 0.081 & 0.102 & $\mathrm{H}_{2} \mathrm{O}(\mathrm{W} 3)$ & 0.080 & 0.116 \\
$\mathrm{H}_{2} \mathrm{O}$ & 0.087 & 0.106 & & & \\
$\mathrm{H}_{2} \mathrm{O}$ & 0.083 & 0.101 & & & \\
$2 \mathrm{C}_{12} \mathrm{O}_{6} \mathrm{H}_{24}$ & 0.108 & - & $2 \mathrm{C}_{12} \mathrm{O}_{6} \mathrm{H}_{24}$ & 0.141 & - \\
\hline
\end{tabular}

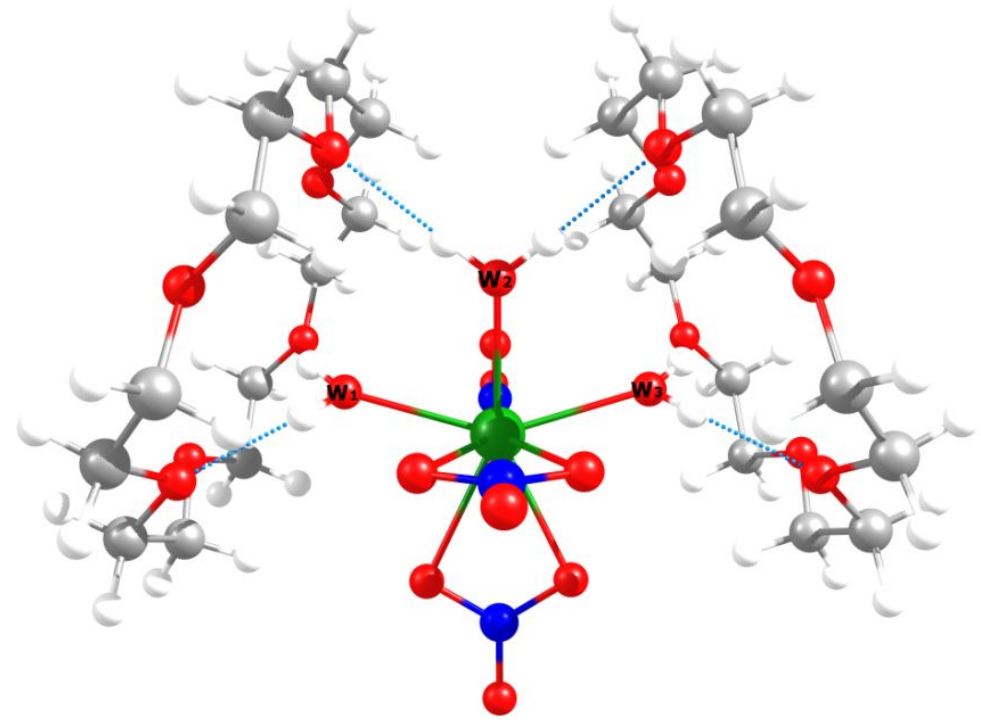

Figure S5. Hydrogen bonds between water ligands coordinated in complex $\mathbf{3}$ with crown ether molecules. Water molecules are labelled according to Table S4 


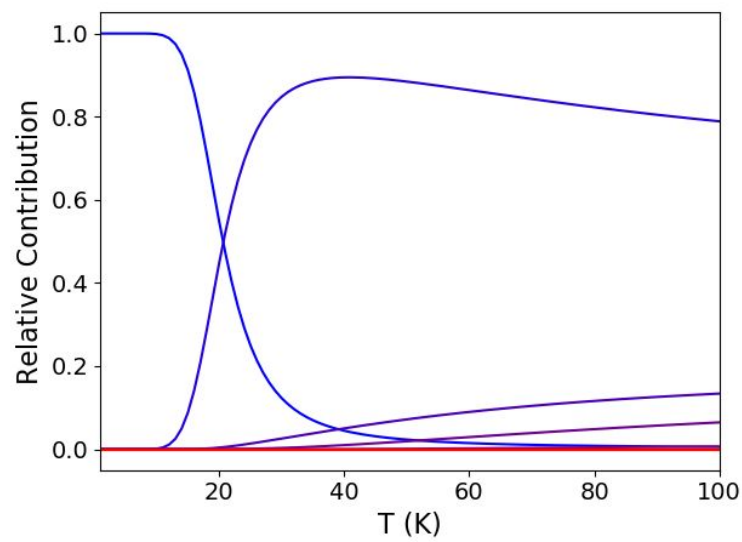

1 'small'

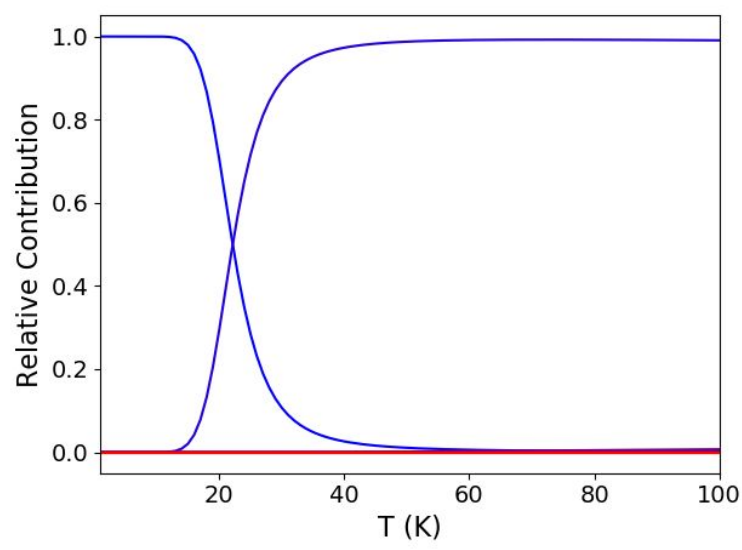

2

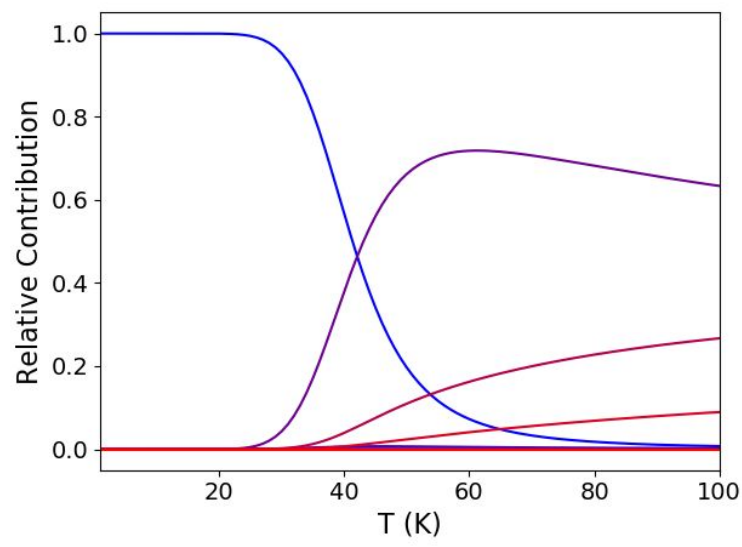

3 'small'

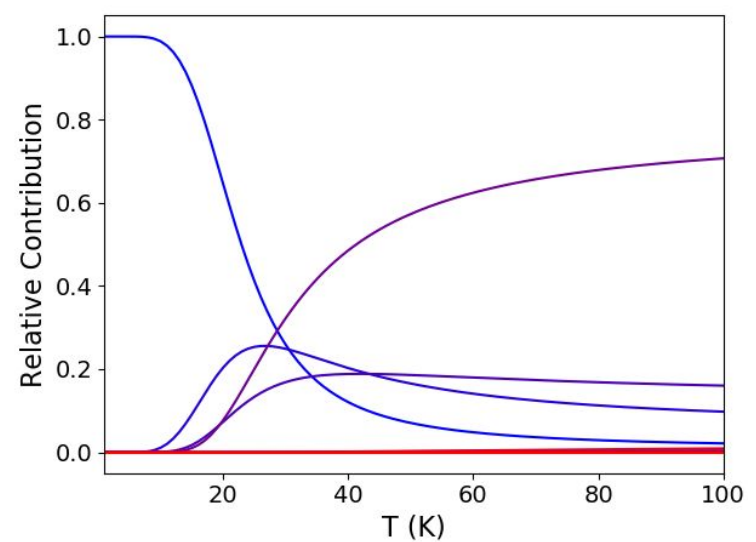

1 'large'

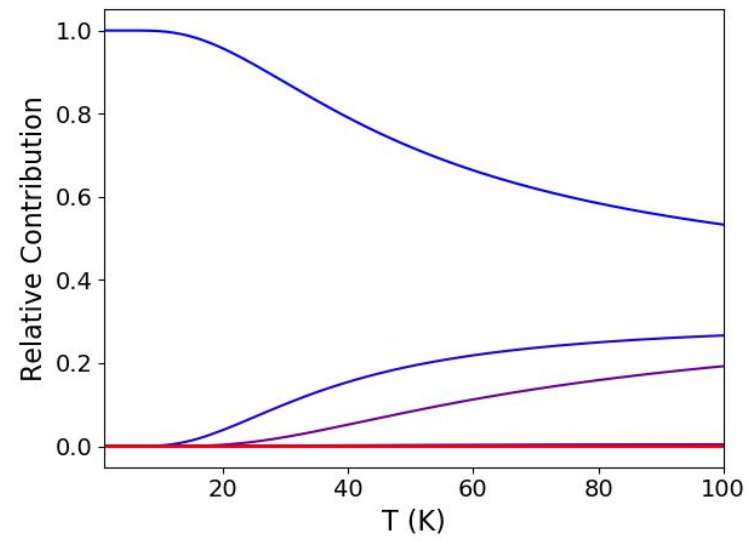

3 'large'

Figure S6. Contribution of the first eight Kramers' doublets to tunnelling demagnetization for 1,2 and 3. First to eighth doublets are depicted in a color scale from blue to red. 
Table S5. Parameters fitted from Cole-Cole plots for $\mathbf{1}$ and $\mathbf{2}$.

\begin{tabular}{|c|c|c|c|c|c|c|c|c|}
\hline Molecule & $\mathrm{T}$ & $\chi_{s, t o t}$ & $\Delta \chi_{1}$ & $\tau_{1}$ & $\alpha_{1}$ & $\Delta \chi_{2}$ & $\tau_{2}$ & $\alpha_{2}$ \\
\hline \multirow[t]{18}{*}{1} & 1.8 & -0.1769 & 5.7989 & $2.07 \mathrm{E}-03$ & 0.6538 & 0.6121 & 0.1582 & 0.0621 \\
\hline & 1.9 & -0.2821 & 5.7664 & $2.01 \mathrm{E}-03$ & 0.6810 & 0.7362 & 0.1513 & 0.1320 \\
\hline & 2.0 & -0.1670 & 5.6243 & $2.60 \mathrm{E}-03$ & 0.6851 & 0.6155 & 0.1384 & 0.1454 \\
\hline & 2.1 & -0.1105 & 5.3848 & 2.69E-03 & 0.6865 & 0.5942 & 0.1281 & 0.1428 \\
\hline & 2.3 & -0.0341 & 5.3608 & $3.63 \mathrm{E}-03$ & 0.6899 & 0.3691 & 0.1837 & 0.1838 \\
\hline & 2.5 & 0.1991 & 4.4938 & 2.69E-03 & 0.6503 & & & \\
\hline & 2.7 & 0.2919 & 4.1370 & $2.52 \mathrm{E}-03$ & 0.6339 & & & \\
\hline & 3.0 & 0.4220 & 3.5801 & 2.13E-03 & 0.6022 & & & \\
\hline & 3.3 & 0.4802 & 3.2126 & $1.70 \mathrm{E}-03$ & 0.5836 & & & \\
\hline & 3.6 & 0.5421 & 2.8549 & $1.37 \mathrm{E}-03$ & 0.5730 & & & \\
\hline & 4.0 & 0.7519 & 2.2941 & $1.29 \mathrm{E}-03$ & 0.5312 & & & \\
\hline & 4.5 & 0.9894 & 1.6816 & $1.25 \mathrm{E}-03$ & 0.4263 & & & \\
\hline & 5.0 & 1.0259 & 1.3677 & 7.74E-04 & 0.3378 & & & \\
\hline & 5.5 & 1.0062 & 1.1741 & $3.94 \mathrm{E}-04$ & 0.2800 & & & \\
\hline & 6.0 & 0.9907 & 0.9713 & $1.78 \mathrm{E}-04$ & 0.2150 & & & \\
\hline & 6.5 & 0.8385 & 0.9922 & $6.77 \mathrm{E}-05$ & 0.2484 & & & \\
\hline & 7.0 & 1.1508 & 0.5533 & 7.22E-05 & 0.0798 & & & \\
\hline & 7.5 & 1.0570 & 0.5487 & $3.19 \mathrm{E}-05$ & 0.0515 & & & \\
\hline \multirow[t]{20}{*}{2} & 1.8 & 0.2991 & 7.5660 & $4.86 \mathrm{E}-01$ & 0.3397 & & & \\
\hline & 1.9 & 0.2856 & 7.3697 & $4.62 \mathrm{E}-01$ & 0.3401 & & & \\
\hline & 2.1 & 0.2624 & 6.7326 & $3.83 \mathrm{E}-01$ & 0.3371 & & & \\
\hline & 2.4 & 0.2431 & 5.6485 & $2.38 \mathrm{E}-01$ & 0.3063 & & & \\
\hline & 2.7 & 0.2333 & 4.7095 & $1.36 \mathrm{E}-01$ & 0.2615 & & & \\
\hline & 3.0 & 0.2252 & 3.9866 & 7.74E-02 & 0.2115 & & & \\
\hline & 3.3 & 0.2140 & 3.4994 & 4.66E-02 & 0.1740 & & & \\
\hline & 3.6 & 0.2022 & 3.1561 & 2.93E-02 & 0.1466 & & & \\
\hline & 3.9 & 0.1902 & 2.8988 & $1.90 \mathrm{E}-02$ & 0.1275 & & & \\
\hline & 4.2 & 0.1792 & 2.6946 & $1.26 \mathrm{E}-02$ & 0.1163 & & & \\
\hline & 4.5 & 0.1693 & 2.5186 & $8.58 \mathrm{E}-03$ & 0.1067 & & & \\
\hline & 5.0 & 0.1574 & 2.2783 & 4.62E-03 & 0.0972 & & & \\
\hline & 5.5 & 0.1514 & 2.0749 & $2.55 \mathrm{E}-03$ & 0.0889 & & & \\
\hline & 6.0 & 0.1513 & 1.8985 & $1.44 \mathrm{E}-03$ & 0.0806 & & & \\
\hline & 6.5 & 0.1536 & 1.7455 & $8.37 \mathrm{E}-04$ & 0.0738 & & & \\
\hline & 7.0 & 0.1565 & 1.6133 & 4.95E-04 & 0.0683 & & & \\
\hline & 8.0 & 0.1605 & 1.3982 & $1.85 \mathrm{E}-04$ & 0.0621 & & & \\
\hline & 9.0 & 0.2252 & 1.1664 & $8.07 \mathrm{E}-05$ & 0.0492 & & & \\
\hline & 10.0 & 0.4332 & 0.8246 & $4.78 \mathrm{E}-05$ & 0.0238 & & & \\
\hline & 11.0 & 0.5288 & 0.6188 & $2.72 \mathrm{E}-05$ & 0.0233 & & & \\
\hline
\end{tabular}

